\title{
Insomnie - Eine Krankheit für Psychiater und Neurologen?
}

Wir Psychiater - ebenso wie unsere neurologischen Kollegen - tun uns seit jeher schwer, Schlafstörungen als ein ernsthaftes klinisches Problem unserer Fachbereiche zu akzeptieren. Das mag daran liegen, dass wir Ein- und Durchschlafschwierigkeiten vor allem als Symptome anderer Diagnosen verstehen, oder weil uns die Störungen nicht als gravierend genug vorkommen. Die Chance, diese Patientengruppe fachärztlich zu behandeln, sollten wir uns jedoch nicht entgehen lassen.

Bereits Ende der 1980er Jahre zeigte die Weltgesundheitsorganisation mit einer weltumfassenden, epidemiologischen Studie den hohen Krankheitswert der chronischen Insomnie auf. Folgestörungen wie schwere Unfälle, Herz-Kreislauf-Erkrankungen, Depression oder Substanzmissbrauch sind die Folgen, mit denen sich nicht nur wir Ärzte sondern die Gesellschaft konfrontiert sehen. Die moderne 24-Stunden-Leistungsund Vergnügungsgesellschaft tut ein Übriges, um die Anzahl der Menschen mit ernsthaften Schlafproblemen zu vermehren. In Deutschland beklagen bereits 4 bis $6 \%$ der Bevölkerung und damit über 4 Millionen Bundesbürger chronische Einund Durchschlafschwierigkeiten, die zu einer Beeinträchtigung der Befindlichkeit und Leistungsfähigkeit am Tage führen. Bei Senioren ist sogar von der doppelten Prävalenzrate auszugehen.
Der Behandlungsbedarf der Betroffenen ist enorm, was an den hohen Verkaufszahlen frei verfügbarer Schlafmittel zu erkennen ist. Bisher haben sich vor allem unsere Hausärzte um diese Patienten gekümmert, nicht schlecht, wie Versorgungsstudien in deutschen Hausarztpraxen zeigen. Die Kollegen konnten dadurch jedoch nicht verhindern, dass sich die Mehrzahl der Patienten unaufhaltsam von einem akut in einen chronisch Kranken entwickeln. Die Behandlung dieser Patienten verlangt fachärztliche Kompetenz. Hier ist die Insomnie bereits die Folge einer komplexen Pathophysiologie, chronifiziert und damit schwer behandelbar und tritt komorbid mit psychischen Krankheiten wie Depression, Angststörungen oder einem Schlafmittelmissbrauch auf.

In dieser Ausgabe werden daher die neurobiologische Pathophysiologie der Insomnie, spezifische gesellschaftliche Auslöser wie Schichtarbeit, Verhaltensempfehlungen zur Prävention von Schlafstörungen und eine kritische Betrachtung der pharmakologischen Behandlungsmöglichkeiten dargestellt. Wenn dies Ihr Interesse für und Ihr Wissen über Ihre Patienten mit Schlafproblemen fördert, dann haben wir mit dem Schwerpunktthema 'Insomnie' unser Ziel erreicht.

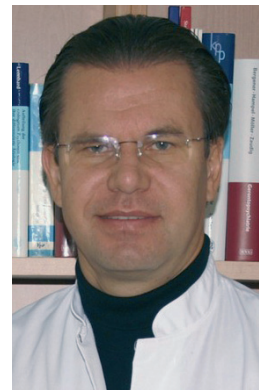

Prof. Dr. med. Göran Hajak 